\title{
A Proposed Hybrid Method for Airtarget Acoustic Signature Diagnosis
}

\author{
Mazhar Taylel ${ }^{1}$, Mahmoud Sabry ${ }^{2}$ \\ EED, Faculty Of Engineering, Alexandria University, Alexandria, Egypt
}

\begin{abstract}
The Airtarget acoustic signal always suffers from noise discrepancies depending on the environmental conditions. It is very useful to use Airtarget Acoustic Signature for detection, classification and recognition process. The merged noise with the acoustic signal differs from place to place and from day time to another. Without de-noising process the results of airtarget captured signal will be inaccurate. Therefore, a proposed preprocessing technique is introduced to analyze a noisy airtarget acoustic signal. Considering the benefits of the discrete wavelet transform in de-noising, it is used to preprocess the AT signature.
\end{abstract}

Keywords: Discrete Wavelet Transform (DWT), Discrete Cosine Transform (DCT), Acoustic Signature (AS), Airtarget (AT), signal to noise ratio (SNR), Signal statistics

\section{Introduction}

Acoustic signature (AS) has a wide usage in Airtarget (AT) detection and classification. It is very useful in detecting very low altitude ATs, which are difficult to be detectable by conventional radars [1]. Its importance arises from its ability to detect ATs that having sudden appearance as drones. Many military researches have been directed to detect ATs using their AS [2]. Generally, systems that use AS for AT detection and classification always suffer from turbulence noise. The most public noise is the wick and wind sound. They present a serious problem at capturing AT acoustic signals [3]. It provides a serious distortion in the analyzed acoustic signal. Therefore, signal de-noising should be used to enhance the AT detection and classification. Using many enhancements processing before signal analysis may cause losses of some information about AT signature. It may cause badness effect on detection and classification accuracy. It also increases the processing time which affect system performance.

Choosing a signal processing method is important to simplify the implementation and provides high accuracy for AT detection and classification. Some signal transformation technique helps in signal enhancement and analysis [4]. The famous transformation method is the wavelet transform. It has a wide usage in signal de-noising [5]. It can be used as $1 \mathrm{D}$ or $2 \mathrm{D}$ transformation. For signal analysis, as acoustic or biomedical signal it works as 1D signal processing, for image processing as image filtering and video coding it produces analysis in 2D of transformation [6]. Also it improves the performance of communication system for ground penetration radar by de-noising the signal [7].

\section{Signal Preprocessing}

The purposed method is to de-noise a received signal and to reconstruct an extracted original signal very closed to the original AS as possible. Almost the entire acoustic signal of ATs in real life suffers from noise like wick and wind noise. It should be preprocessed before being analyzed. The present work deals with a noisy AT signal analysis for detection and classification. As the noise increases in AS a hardness of feature extraction exists. The noise usually has low amplitude and high frequency where AS has high amplitude with low frequency. The received AT signal can be represented by the following equation:

$$
\mathrm{X}[\mathrm{n}]=\mathrm{S}(\mathrm{n})+\mathrm{N}(\mathrm{n})
$$

where $X[n]$ is the received signal, $S[n]$ is an input original signal and $\mathrm{N}[\mathrm{n}]$ is noise combined with the signal.

A filtration process should be included in the proposed system. In the present system the DWT is used in processing stages. Due to its benefits in filtration, it has been used to recover the original AT-AS of the captured acoustic signal with noise for multilevel analysis and signal statistics.

The purposed method is to de-noise a received signal and to reconstruct an extracted original signal very closed to the original AS as possible. Almost the entire acoustic signal of ATs in real life suffers from noise like wick and wind noise. It should be preprocessed before being analyzed. The present work deals with a noisy AT signal analysis for detection and classification. As the noise increases in AS a hardness of feature extraction exists.

Noise usually has low amplitude and high frequency where AS has high amplitude with low frequency. The received AT signal can be represented by the following equation:

$$
X[n]=S(n)+N(n)
$$

where $X[n]$ is the received signal, $S[n]$ is an input original signal and $\mathrm{N}[\mathrm{n}]$ is noise combined with the signal.

A filtration process should be included in the proposed system. In the present system the DWT is used in processing stages. Due to its benefits in filtration, it has been used to recover the original AT-AS of the captured acoustic signal with noise for multilevel analysis and signal statistics. 


\section{International Journal of Science and Research (IJSR) \\ ISSN (Online): 2319-7064}

Index Copernicus Value (2013): 6.14 | Impact Factor (2015): 6.391

\section{Proposed Hybrid System}

The Proposed Hybrid System (PHS) deals with capturing of AT acoustic signal. The PHS consists of two main channels: Preprocessing channel and feature extraction channel as shown in figure (1).

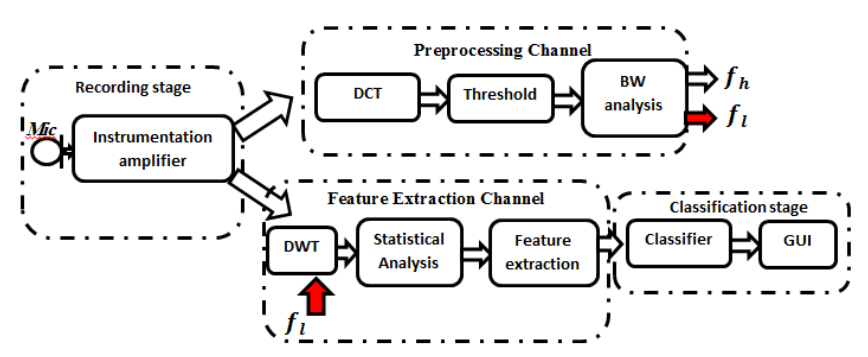

Figure 1: The PHS block diagram.

\subsection{Recording Stage}

The first stage is a recording stage consisting of a dynamic sensitive microphone to capture the AT acoustic signal from the field at run time. The microphone is characterized by high sensitivity $10-50 \mathrm{mV} / \mathrm{Pa}$ followed by an instrumentation amplifier.

\subsection{Preprocessing Channel}

A preprocessing channel is the second stage in the PHS. The main objective from the preprocessing channel is to make a decision for the stopping level of the DWT process and ensures that a suitable sampling rate is selected for this end level. The DCT is used to get the spectral power density (SPD) of the amplified captured AS to determine a $-3 \mathrm{~dB}$ threshold value and to define the lowest frequency $\left(f_{l}\right)$, highest frequency $\left(f_{h}\right)$, BW and the allowable DWT (L) levels of the AT-AS feature extraction.

The highest frequency of the AS-BW is used to ensure that the sampling rate of the recording system is the most suitable for digital signal processing to satisfy the Nyquest rule.

$$
f_{\text {sampling }} \geq 2 f_{h}
$$

where $\left(\boldsymbol{f}_{\text {sampling }}\right)$ is the sampling frequency of analogue to digital converter of the recording system.

The $f_{l}$ of the BW is used to determine the number of DWT (L) levels for the captured AS.

$$
L=\operatorname{In} t\left(\left(1.4427 * \ln \left(\frac{f_{\text {sampling }}}{f_{l}}\right)\right)-1\right)
$$

\subsection{Feature Extraction Channel}

The feature extraction is the second main channel in the PHS. It includes of three processes:

DWT, signal statistical analysis and feature extraction. The DWT analyzes the AS up to the (L) level within the selected BW $\left(f_{l}, f_{h}\right)$. The approximation coefficients of the multiple (L) levels are evaluated using the signal statistics tools:
Mean, Variance, Median and Entropy to extract the main features characterizing the AT acoustic signal.

\subsection{Classification Stage}

A classifier based on the K-Nearest Neighbor (KNN) is to be used. It uses the extracted features of AS to classify the AT captured signal. The GUI stage is used to show the output results of the classifier as an interface between the PHS and the user.

\section{System Verification}

To simulate the PHS F-16 aircraft acoustic signal has been downloaded from internet as an example as follows:

\subsection{The F-16 Original Signal Analysis}

According to the sequence of signal processing in the PHS, the F-16 AS sample will pass through the preprocessing channel to determine the $\left(f_{l}, f_{h}\right), \mathrm{BW}$ and $\mathrm{L}$.

The F-16 AS- SPD is analyzed using the DCT as shown in figure (2).

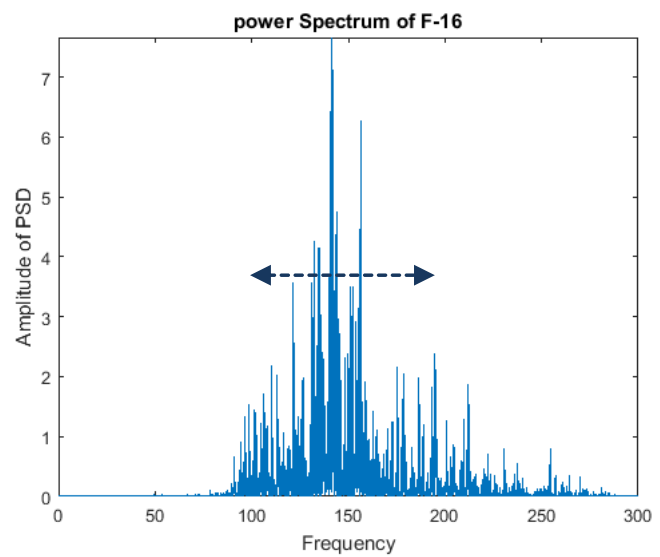

Figure 2: The SPD of F-16 aircraft AS.

The threshold process in the preprocessing channel uses the $3 \mathrm{~dB}$ value as a criterion to determine $\left(f_{l}, f_{h}\right)$ and $\mathrm{BW}$ of the $\mathrm{F}$ 16 AS. The sampling rate of the downloaded sample for F$16 \mathrm{AS}$ is $11025 \mathrm{~Hz}$. It is found that the threshold level is at $3.83 f_{l}=132.3 \mathrm{~Hz}, f_{h}=156.4 \mathrm{~Hz}$, the $\mathbf{B W}=24.1 \mathrm{~Hz}$ and the peak amplitude of the F-16 AS is at $141.5 \mathrm{~Hz}$.

By using equation (3) the calculated stopped level of the DWT analysis will be the fifth level.

From these results the feature extraction channel is to be begun for achieving its objective. The AS statistical analysis is being applied to the approximation coefficients of the DWT five levels.

The output is tested by measuring the SNR for the original F-16 AS. It is found that the SNR of the zero-level is equal to $\mathbf{- 1 2 . 0 6} \mathbf{~ d B}$ whereas for the fifth level is equal to 10.012 $\mathbf{~ d B}$ at the fifth level. As shown in figure (3) by the solid blue line. It is seen that the SNR increases with the level increase up to the selected level of the DWT-BW for the tested AT-AS. Table (1) shows the results of signal 


\section{International Journal of Science and Research (IJSR) \\ ISSN (Online): 2319-7064}

Index Copernicus Value (2013): 6.14 | Impact Factor (2015): 6.391

statistics on the approximation coefficients of the F-16 AS up to the fifth level.

Table 1: The F-16 acoustic signal statistics

\begin{tabular}{|c|c|c|c|c|}
\hline DWT levels & Mean & Median & Variance & Entropy \\
\hline Level 1 & 0.016286 & 0.0356193 & 0.035491 & 4.604269 \\
\hline Level 2 & 0.023023 & 0.0468731 & 0.049252 & 4.826551 \\
\hline Level 3 & 0.032536 & 0.0838753 & 0.087104 & 5.048071 \\
\hline Level 4 & 0.045939 & 0.1616917 & 0.167181 & 5.168349 \\
\hline Level 5 & 0.064780 & 0.2505101 & 0.262907 & 5.255075 \\
\hline
\end{tabular}

\subsection{Noisy Signal Analysis for F-16 aircraft}

A wind acoustic signal has been merged with the tested sample of the F-16 AS for studying the performance of the proposed PHS. The SNR has been measured for the noisy F-16 AS and it is found for the zero level is equal to-12.96 $\mathrm{dB}$ and at the fifth level was equal to $-11.667 \mathrm{~dB}$ as shown in figure (3) by the dashed red line.

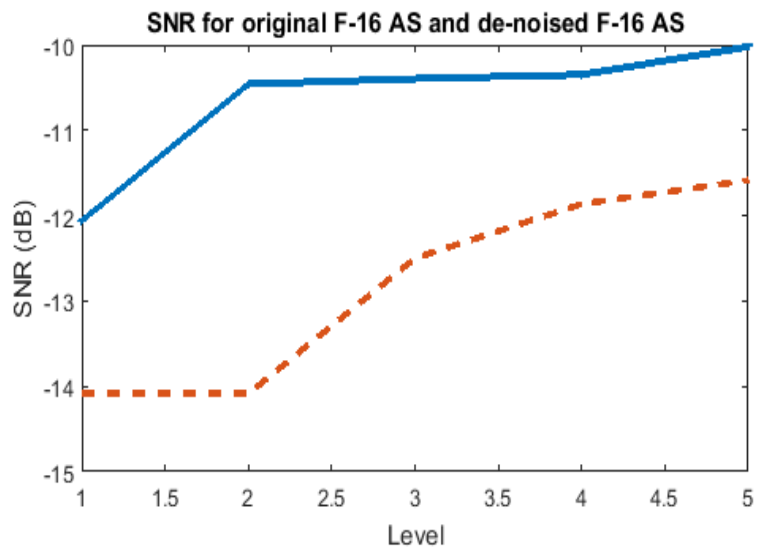

Figure 3: The measured SNR for the original F-16 AS and the de-noised F-16 AS

Table (2) shows the results of the signal statistics of the merged acoustic signal up to the fifth level.

Table 2: The F-16 + wind for noisy AS statistics

\begin{tabular}{|c|c|c|c|c|}
\hline DWT levels & Mean & Median & Variance & Entropy \\
\hline Level 1 & 0.016521 & 0.0150580 & 0.050073 & 4.618930 \\
\hline Level 2 & 0.023358 & 0.0217245 & 0.099199 & 4.847366 \\
\hline Level 3 & 0.033012 & 0.0306344 & 0.149969 & 5.010394 \\
\hline Level 4 & 0.046628 & 0.0455395 & 0.213297 & 5.164855 \\
\hline Level 5 & 0.065782 & 0.0639236 & 0.379588 & 5.153133 \\
\hline
\end{tabular}

\section{Signal Reconstruction}

By comparing the results of tables (1) and (2) it is found that the difference is too small at Mean, Variance, Median and Entropy as shown in figure (4). The statistical results of the original F-16 AS are plotted by the red line and the black line shows the statistical results of the de-noised F-16 AS. (a)
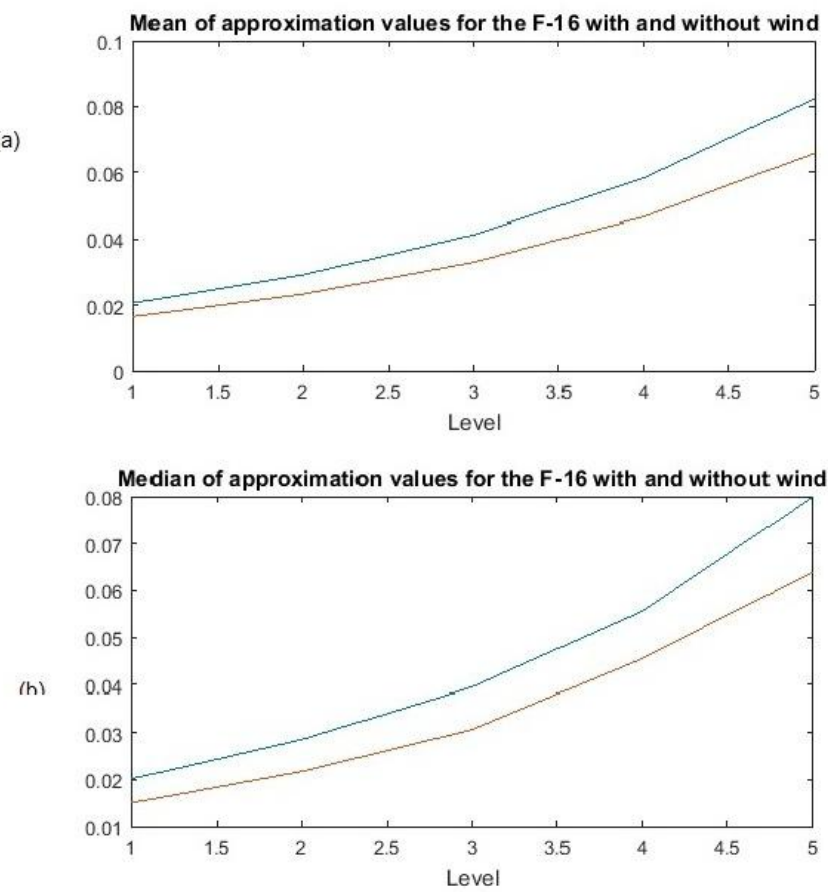

(c)

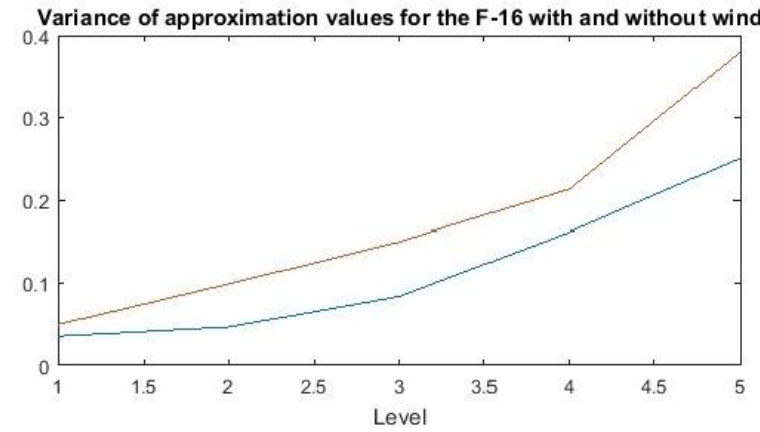

(d)

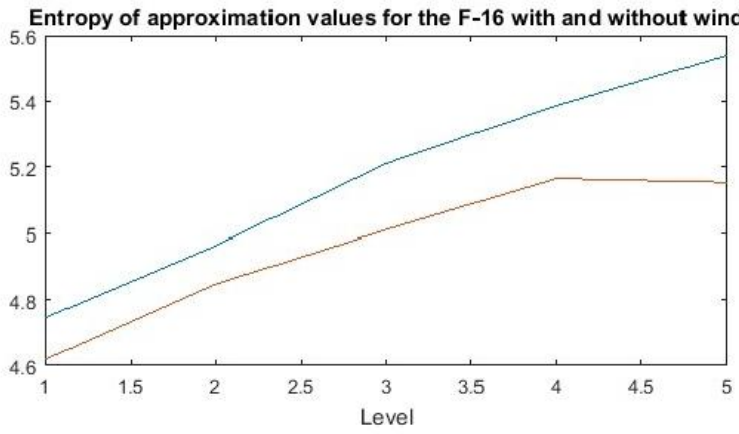

Figure (4): The comparison among statistical analysis for the original F-16 AS and the de-noised F-16 AS

Another comparing between the F-16 original AS and the F16 reconstructed noisy AS the variance has been measured. Table (3) and figuer (5) show the variance for (Mean, Variance, Median and Entropy).

Table 3: Comparison among the statistical parameters

\begin{tabular}{|c|c|c|c|c|}
\hline \multirow{2}{*}{$\begin{array}{c}\text { DWT } \\
\text { Levels }\end{array}$} & Mean & Variance & Median & Entropy \\
\cline { 2 - 5 } Level 1 & $8.5593 \mathrm{E}-06$ & $1.0445 \mathrm{E}-04$ & $1.2998 \mathrm{E}-05$ & $8.0217 \mathrm{E}-03$ \\
\hline Level 2 & $1.7124 \mathrm{E}-05$ & $1.3690 \mathrm{E}-03$ & $2.2979 \mathrm{E}-05$ & $6.6782 \mathrm{E}-03$ \\
\hline Level 3 & $3.4264 \mathrm{E}-05$ & $2.1842 \mathrm{E}-03$ & $3.9543 \mathrm{E}-05$ & $2.0094 \mathrm{E}-02$ \\
\hline Level 4 & $6.8619 \mathrm{E}-05$ & $1.3316 \mathrm{E}-03$ & $5.0717 \mathrm{E}-05$ & $2.4553 \mathrm{E}-02$ \\
\hline Level 5 & $1.3749 \mathrm{E}-04$ & $8.3306 \mathrm{E}-03$ & $1.2817 \mathrm{E}-04$ & $7.4642 \mathrm{E}-02$ \\
\hline
\end{tabular}

Volume 5 Issue 6, June 2016 www.ijsr.net 


\section{International Journal of Science and Research (IJSR)}

ISSN (Online): 2319-7064

Index Copernicus Value (2013): 6.14 | Impact Factor (2015): 6.391

(a)

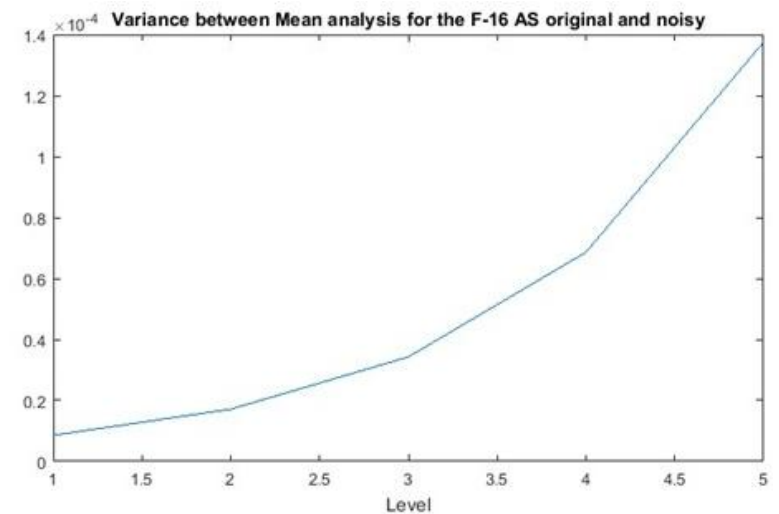

(b)

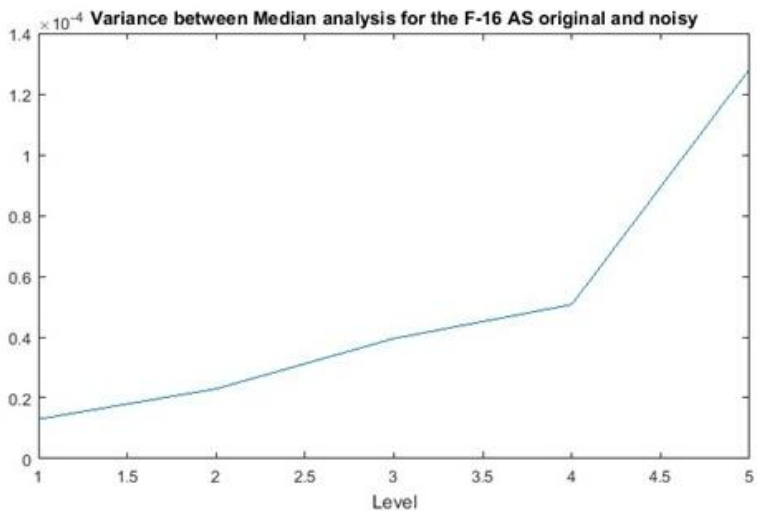

(c)

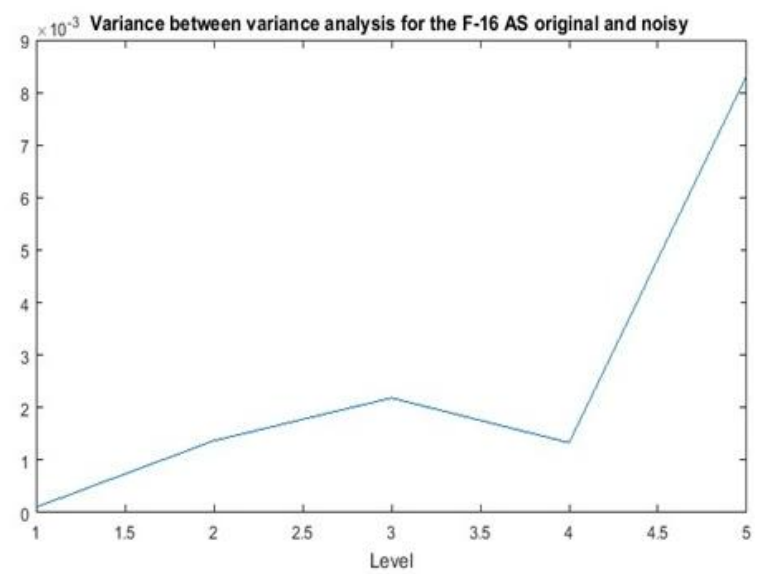

(d)

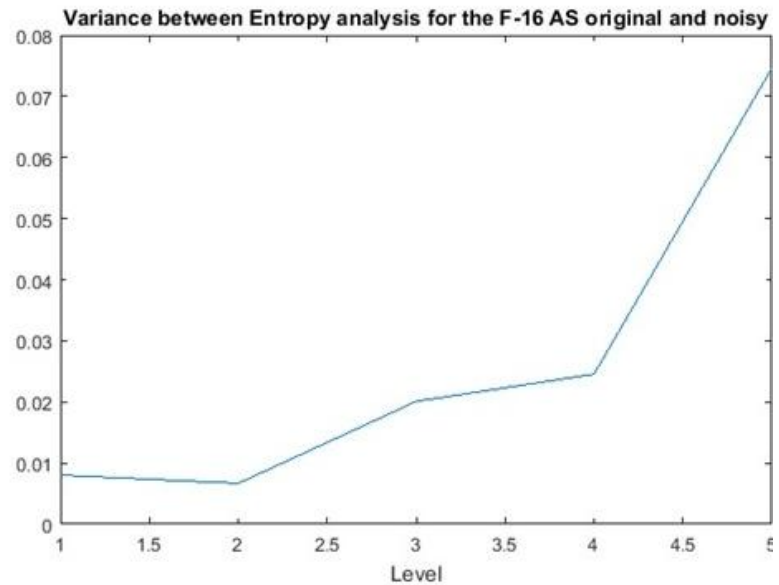

Figure 5: The variance for the statistical parameters of the original F-16 original AS and the de-noising F-16 AS.

\section{Results}

The comparison between the Statistical parameters results for the original F-16 AS and the de-noising F-16 AS is achieved by using the SNR and the variance measurements. It is found that the results of the variance values at the DWT five levels was too small as shown in table (3). The difference between the SNR at the DWT fifth levels was equal to $-1.5639 \mathrm{~dB}$. All of this results provides a success for the using the PHS in signal reconstruction with high performance.

\section{Conclusion}

The Proposed Hybrid System is characterized by two processing channels. The first channel is used to determine values of $f_{l}, f_{h}$, bandwidth and the allowable levels of the acoustic signature to be used in the discrete wavelet transform. The second channel is level limited analysis to extract the main features of the acoustic signature to be used in the classification process. From the above discussion it is seen that the Proposed Hybrid System provides high performance in the noisy surrounding. This benefit is shown from the difference between the measured SNR values for the original F-16 AS and the de-noised F-16 AS at the DWT fifth level was equal to $\mathbf{- 1 . 5 6 3 9} \mathbf{~ d B}$.

\section{References}

[1] Weiqun Shi, Gus Arbadjis, Brett Bishop, Peter Hill, Rich Plasse, and Yoder, "Detecting, Tracking and Identifying Airborne Threats with Netted Sensor Fence". In C. Thomas (Ed.), Sensor Fusion - Foundation and Applications, 2011

[2] Victor pratap singh and prof. phalguni Gupta "passive acoustic localization (prototype designe and implementation for military application), M.tech thises report y6111048 submitted in june 2008

[3] Gaetano Caronna, Ivan Roselli, Pierluigi Testa ," Acoustic System for Aircraft Detection and Tracking based on Passive Microphones Arrays ", 148th Meeting of the Acoustical Society of America San Diego, November 2004

[4] Matz V., Kreidl, M., Šmíd, R.: Signal-to-Noise Ratio Improvement based on the Discrete Wavelet Transform in Ultrasonic Defectoscopy. Acta Polytechnica. 2004, vol. 44, no. 4, s. 61-66. ISSN 1210-2709

[5] Mazhar Tayel, Mahmoud Sabry "A Detection and Identification Method for Airtarget Acoustic Signal Characterization" IOSR Journal of VLSI and Signal Processing (IOSR-JVSP) Volume 6, Issue 2, Ver. I (Mar. Apr. 2016), PP 21-24.

[6] Rajeev Aggarwal , Jai Karan Singh, Vijay Kumar Gupta, Sanjay Rathore, Mukesh Tiwari and Dr. Anubhuti Khare " Noise Reduction of Speech Signal using Wavelet Transform with Modified Universal Threshold " International Journal of Computer Applications (0975 8887) Volume 20- No.5, April 2011

[7] Baili J., Lahouar S., Hergli M., Amimi A. and Besbes K., "Application of the Discrete Wavelet Transform to Denoise GPR Signals," in proceedings of ISCCSP, 2006. 